\title{
Determinação da Idade de Transição entre Lenho Juvenil e Lenho Adulto para Três Espécies Florestais por meio de suas Propriedades Mecânicas
}

\author{
Tainise Vergara Lourençon ${ }^{1}$, Bruno Dufau Mattos ${ }^{1 \star}$, Darci Alberto Gatto ${ }^{2}$, \\ Ediane Andréia Buligon ${ }^{3}$, Clóvis Roberto Haselein ${ }^{3}$ \\ ${ }^{1}$ Centro de Desenvolvimento Tecnológico, Universidade Federal de Pelotas - UFPel, Pelotas/RS, Brasil \\ ${ }^{2}$ Centro de Engenharias, Universidade Federal de Pelotas - UFPel, Pelotas/RS, Brasil \\ ${ }^{3}$ Centro de Ciências Rurais, Universidade Federal de Santa Maria - UFSM, Santa Maria/RS, Brasil
}

\begin{abstract}
RESUMO
Objetivou-se definir a idade de transição entre o lenho juvenil e o lenho adulto das madeiras de Luehea divaricata (açoita-cavalo), Platanus $\times$ acerifolia (plátano) e Carya illinoinensis (nogueira-pecã), através de MOE e MOR em flexão estática. Para tanto, foram confeccionados corpos de prova no sentido radial e realizaram-se os ensaios de flexão estática em máquina universal de ensaios. Para determinação da idade de transição dos lenhos, os dados foram plotados no sentido radial, individualmente para cada árvore, e uma regressão foi utilizada para estimar a variação dos dados de forma geral e outra regressão linear foi utilizada na parte ascendente dos pontos. O limite da parte crescente dos pontos determinou a zona de transição entre os lenhos juvenil e adulto. De acordo com os resultados, foi observado que não houve diferença na idade de transição, para cada espécie, em relação à propriedade (MOE e MOR) utilizada para delimitar a zona de transição. Obteve-se idade média de transição entre lenhos de 9,3 para a madeira de plátano, 11,8 para nogueira-pecã e 23,8 para açoita-cavalo.
\end{abstract}

Palavras-chave: MOE, MOR, açoita-cavalo, plátano, nogueira-pecã.

\section{Determination of the Age of Transition from Juvenile to Mature Wood for Three Forest Species by Mechanical Properties}

\begin{abstract}
The aim of present study was to define the age of segregation of the woods of Luehea divaricata, Platanus x acerifolia and Carya illinoinensis, by Modulus of Rupture (MOR) and Modulus of Elasticity (MOE). To this end, test specimens were prepared in the radial direction and static bending tests were carried out in universal testing machine. To determine the zone of transition between juvenile and mature wood, the data were plotted in the radial direction individually for each tree, a regression was used to estimate the variation of data in general, and another linear regression was used at the initial linear points. The boundary of the increasing part of the data determined the transition zone between juvenile and mature wood. The results showed that there was no difference in age of transition, for each species, in relation to the property (MOR and MOE) used to delimit the transition zone. The following average ages of transition were obtained: 9.3 years for Platanus x acerifolia, 11.8 years for Carya illinoinensis and 23.8 years for Luehea divaricata.
\end{abstract}

Keywords: MOE, MOR, Luehea divaricata, Platanus x acerifolia, Carya illinoinensis. 


\section{INTRODUÇÃO}

Segundo Evangelista et al. (2010), a importância do conhecimento da variabilidade interna das propriedades da madeira está ligada ao seu melhor uso tecnológico, visando à classificação da madeira serrada com propriedades semelhantes, bem como à homogeneização de lotes para a sua comercialização. A correta utilização desse material somente será efetiva mediante o completo conhecimento de suas diferentes propriedades, consequência de sua constituição química e, principalmente, anatômica.

Sabe-se que a variabilidade das propriedades da madeira é consequência de diferenças estruturais, desde a nanoestrutura da parede celular até as geográficas. A fonte de variação anatômica da árvore talvez seja a mais significativa, sendo a variação no sentido radial a mais importante. A extensão desta variação é, principalmente, determinada pela presença da madeira juvenil, sua proporção no tronco e suas características físico-químicas e anatômicas (Leonello et al., 2008).

Assim, a madeira pode ser dividida, no sentido radial, entre lenho juvenil, próximo à medula, e lenho adulto, próximo à casca. Esse desenvolvimento caracteriza-se por mudanças progressivas no câmbio e, consequentemente, nas dimensões das células por este geradas, o que influencia nas propriedades da madeira, como a massa específica básica (Panshin \& de Zeeuw, 1964; Zobel \& Buijtenen, 1989).

Segundo Bendtsen (1978), as propriedades da madeira de coníferas de rápido crescimento não são uniformes da medula para o exterior; a madeira dos primeiros anéis formados apresenta menor massa específica, menor comprimento de fibra e maior ângulo microfibrilar, com rápidas taxas de mudanças nas propriedades, entre outras características. Em anéis sucessivos, partindo do centro da árvore, verifica-se o aumento gradativo da massa específica e das características anatômicas (comprimento, diâmetro do lume e espessura das paredes das fibras etc.), caracterizando a transição do lenho juvenil para o lenho adulto.

Comparando-se o lenho adulto e o lenho juvenil de espécies de coníferas de rápido crescimento, o lenho juvenil se difere por apresentar uma massa específica mais baixa, menor ângulo microfibrilar, traqueídeos mais curtos, menor contração transversal, maior contração longitudinal, maior proporção de lenho de reação, menor porcentagem de lenho tardio, maior conteúdo de lignina, menor conteúdo de celulose e menor resistência mecânica (Latorraca \& Albuquerque, 2000; Larson et al., 2001).

O’Neill \& Tarigo (2003), ao estudarem valores de resistência mecânica da madeira de Eucalyptus grandis, $P$. taeda e $P$. elliottii, observaram valores menores próximo à medula (madeira juvenil) e maiores, próximo à casca (madeira adulta), exceto para o eucalipto. Para os mesmos autores, a obtenção de árvores com maiores proporções de madeira adulta representa um ciclo mais longo de corte e permite maiores volumes de madeira apta para uso estrutural.

Para a caracterização das propriedades mecânicas da madeira, são utilizados o módulo de ruptura (MOR) e o módulo de elasticidade (MOE), em método destrutivo de flexão estática, o qual consiste em uma carga aplicada tangencialmente aos anéis de crescimento até a ruptura. Essa propriedade é relacionada à qualidade da madeira, sendo muito utilizada em trabalhos de pesquisa na área de tecnologia de produtos florestais. Todavia, ainda que, por propriedades anatômicas e até propriedades físicas, existam estudos que definam a idade de transição entre lenhos juvenil e adulto para muitas madeiras, não se tem comprovação científica se o MOE e o MOR são bons parâmetros para este tipo de estimativa.

Larson et al. (2001) comentaram que o MOR e o MOE são influenciados pelo percentual de lenho juvenil presente na madeira de espécies de pinus. Os autores relataram que existem poucos estudos comparativos entre estas propriedades nos dois tipos de lenho. Eles ainda descrevem que os módulos de flexão estática são altamente correlacionados com a massa específica, com o comprimento de fibra e o ângulo fibrilar, pois a fibra é considerada a fonte de elasticidade e resistência da madeira.

O objetivo do presente trabalho foi determinar a idade de transição entre o lenho juvenil e o lenho adulto, por meio de MOE e MOR em flexão estática, de três espécies florestais de folhosas. 


\section{MATERIAL E MÉTODOS}

Para o estudo de determinação da idade de transição do lenho juvenil para o lenho adulto através de propriedades mecânicas, foram selecionadas cinco árvores para cada espécie: Platanus $\times$ acerifolia (Ait.) Wild. (plátano), Luehea divaricata Mart. Et Zucc (açoita-cavalo) e Carya illinoinensis (Wangenh) K. Koche (nogueira-pecã), provenientes da Região Central do Rio Grande do Sul. O procedimento de seleção foi realizado por extração ao acaso, conforme norma estabelecida pela COPANT (1971), e as características dos indivíduos selecionados estão apresentadas na Tabela 1.

Das árvores selecionadas, foi retirada a primeira tora, com comprimento de $2,5 \mathrm{~m}$; desta, retirou-se um pranchão central orientado no sentido longitudinal com $8 \mathrm{~cm}$ de espessura. Posteriormente, foram preparadas amostras de $40 \mathrm{~cm}$ de comprimento retiradas da base do pranchão e destas foram confeccionados corpos de prova de $2 \times 2 \times 40 \mathrm{~cm}$
(ASTM, 2000), no sentido medula-casca, conforme procedimento mostrado na Figura 1.

Os corpos de prova foram transportados até o Laboratório de Produtos Florestais (LPF) da UFSM, onde permaneceram em câmara climatizada a $20^{\circ} \mathrm{C} \mathrm{e}$ $65 \%$ de umidade relativa do ar, por aproximadamente 60 dias. Estabilizado o teor de umidade da madeira a $12 \%$, realizaram-se os ensaios mecânicos.

Os ensaios de flexão estática nos corpos de prova biapoiadados com carregamento no meio do vão foram realizados em máquina universal de ensaios, hidráulica, marca AMSLER (dotada de programa de aquisição automática de dados), com célula de carga de $20 \mathrm{t}$, a uma velocidade de deformação de 1,3 mm/ min.

Para a determinação da idade de transição do lenho juvenil para o lenho adulto, utilizouse uma adaptação da metodologia de análise de dados proposta por Ferreira et al. (2011). Para este procedimento, os resultados das propriedades mecânicas no sentido radial para cada árvore foram plotados, a zona linear da curva foi separada

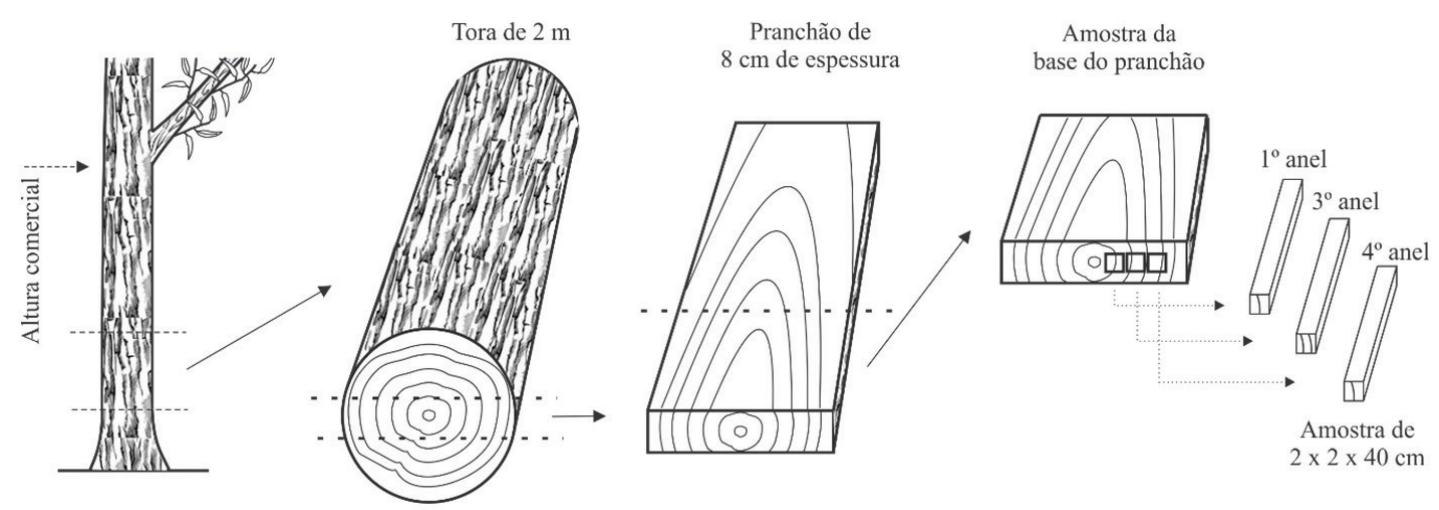

Figura 1. Confecção dos corpos de prova no sentido medula-casca para o estudo de delimitação do lenho juvenil e lenho adulto.

Figure 1. Confection of the wood samples from pith to bark for the study of delimiting the juvenile and mature wood zones.

Tabela 1. Dados médios para diâmetro à altura do peito (DAP), altura e idade das três espécies utilizadas. Table 1. Mean value for diameter at breast height (DBH), height and age for three woods.

\begin{tabular}{|c|c|c|c|c|c|}
\hline \multirow{2}{*}{ Espécie } & \multirow{2}{*}{$\operatorname{DAP}(\mathrm{cm})$} & \multicolumn{2}{|c|}{ Altura (m) } & \multirow{2}{*}{ Idade (anos) } & \multirow{2}{*}{$\begin{array}{c}\text { Espaçamento de } \\
\text { plantio (m) }\end{array}$} \\
\hline & & Comercial & Total & & \\
\hline Plátano & 35,6 & 12,4 & 18,8 & 23,2 & $3,0^{*}$ \\
\hline Açoita-cavalo & 39 & 8,8 & 15,7 & 49,2 & Mata nativa ${ }^{* *}$ \\
\hline Nogueira-pecã & 34,8 & 5,7 & 12,1 & 18,8 & $3,0 \times 3,0^{* * *}$ \\
\hline
\end{tabular}

* Árvores oriundas de plantios para quebra-vento. ${ }^{* *}$ Processo licenciado pela Secretaria Estadual do Meio Ambiente (Rio Grande do Sul). ${ }^{* *}$ Árvores oriundas de plantios para frutos. 
e realizou-se uma nova regressão. Ao término da região linear do gráfico, delimitada pela regressão linear, traçou-se uma perpendicular ao eixo referente à idade (plotado como anéis de crescimento) e assim determinou-se a idade em que ocorre a transição do lenho juvenil para o lenho adulto, por meio do comportamento do módulo de elasticidade (MOE) e do módulo de ruptura (MOR), ao longo do sentido radial. Os modelos das curvas de regressão foram estabelecidos considerando-se $5 \%$ de probabilidade de erro.

\section{RESULTADOS E DISCUSSÃO}

As Figuras 2, 3 e 4 apresentam os resultados do módulo de elasticidade (MOE) e do módulo de ruptura (MOR) no sentido medula-casca, em função dos anéis de crescimento, utilizados para a delimitação dos lenhos juvenil e adulto das madeiras de açoita-cavalo, nogueira-pecã e plátano, respectivamente. Os resultados foram separados por árvores conforme os procedimentos utilizados por Ferreira et al. (2011).

De maneira geral, para as três espécies, tanto o módulo de elasticidade quanto o módulo de ruptura apresentaram comportamento crescente seguido de uma posterior estabilização ao longo do sentido medula-casca. Todavia, em alguns casos, o comportamento das propriedades mecânicas foi crescente, estável e decrescente ao longo do sentido radial, como foi o caso das árvores 4 e 5 de nogueirapecã, 2 e 4 de açoita-cavalo e da árvore 5 de plátano.

Assim como verificado no presente estudo, Lima \& Garcia (2011), ao estudarem a variação das propriedades mecânicas de Eucalyptus grandis no sentido medula-casca, observaram que o MOE em flexão estática cresce e logo se estabiliza quando se aproxima das regiões próximas à casca; todavia, para o MOR, os autores encontraram valores em ordem crescente desde a medula até a casca, diferindo ligeiramente do observado no presente estudo.

Por outro lado, diferentemente do observado para as madeiras deste estudo, Gonçalves et al. (2009) verificaram comportamento linear e crescente para o MOE e o MOR da madeira de híbridos clonais de Eucalyptus urophylla $\times$ Eucalyptus grandis. No entanto, o material arbóreo utilizado no estudo de Gonçalves et al. (2009) apresentava idade em torno de 70 meses; assim, acredita-se que esse comportamento crescente ao longo de todo sentido radial se dê pela ausência da formação de lenho adulto, o qual teoricamente estabilizaria as propriedades mecânicas.

A estabilização das propriedades mecânicas ao longo do sentido radial, tanto para as madeiras do presente estudo quanto para as utilizadas como referência literária, dá-se principalmente pela modificação dos constituintes anatômicos, em que as fibras tendem a aumentar o comprimento, o diâmetro e a espessura de parede enquanto o lume tende a diminuir (Gatto et al., 2007, 2008, 2010; Ferreira et al., 2011; Lobão et al., 2012), tornando a estrutura da madeira mais rígida e resistente.

Por meio das Figuras 2, 3 e 4, observou-se que ocorreu uma ligeira variação, dentro de cada árvore, na idade de transição entre os lenhos juvenil e adulto, estimada pelo MOE e pelo MOR para as espécies de plátano, nogueira-pecã e açoita-cavalo. Assim, utilizou-se a análise de variância fatorial (ANOVA fatorial), para averiguar se a delimitação efetuada pelo MOE ou pelo MOR apresenta resultados estatisticamente diferentes (Tabela 2).

Tabela 2. Análise de variância fatorial para a idade de transição em função da espécie e da propriedade (MOE e MOR).

Table 2. Factorial analysis of variance for the transition age as a function of wood specie and mechanical properties.

\begin{tabular}{cccccc}
\hline Propriedade & Fator & SQ & Gl & QM & Razão F \\
\cline { 2 - 6 } & Espécie (A) & 1199,65 & 2 & 599,83 & $83,78^{* *}$ \\
Idade de transição & Propriedade (B) & 0,3 & 1 & 0,3 & $0,04^{\text {ns }}$ \\
\cline { 2 - 6 } & A $\times$ B & 12,44 & 2 & 6,22 & $0,87^{\text {ns }}$ \\
\hline
\end{tabular}

$\mathrm{SQ}=$ soma dos quadrados; $\mathrm{Gl}$ = grau de liberdade, $\mathrm{QM}=$ quadrado médio; ${ }^{* *}$ significativo em nível de $1 \%$ de probabilidade de erro; ns = não significativo. 

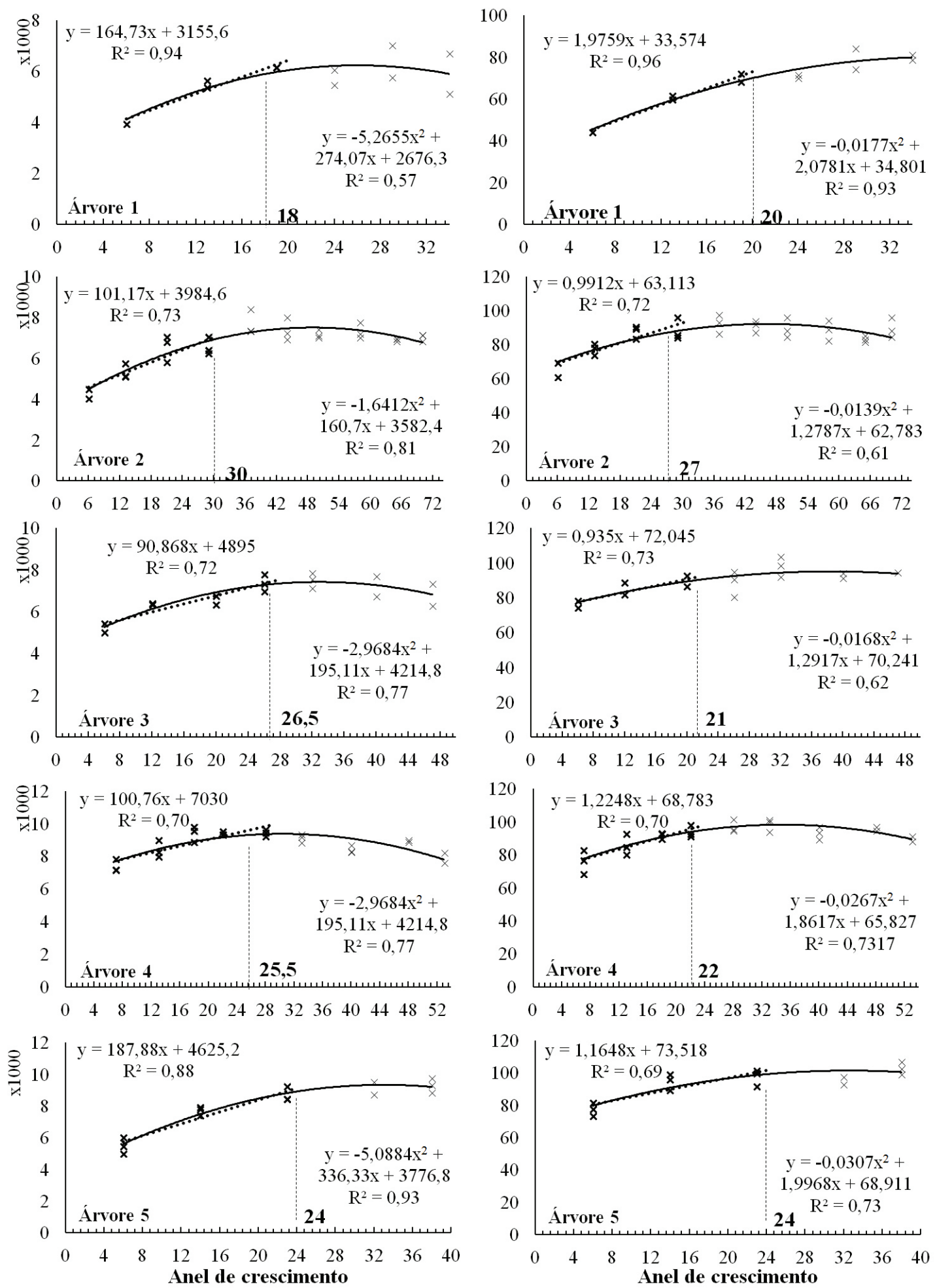

Figura 2. Variação do MOE (esquerda) e MOR (direita), e demarcação de lenhos juvenil e adulto para as cinco árvores de açoita-cavalo.

Figure 2. Variation of MOE (left) and MOR (right) and demarcation juvenile and mature zones for the five açoitacavalo trees. 

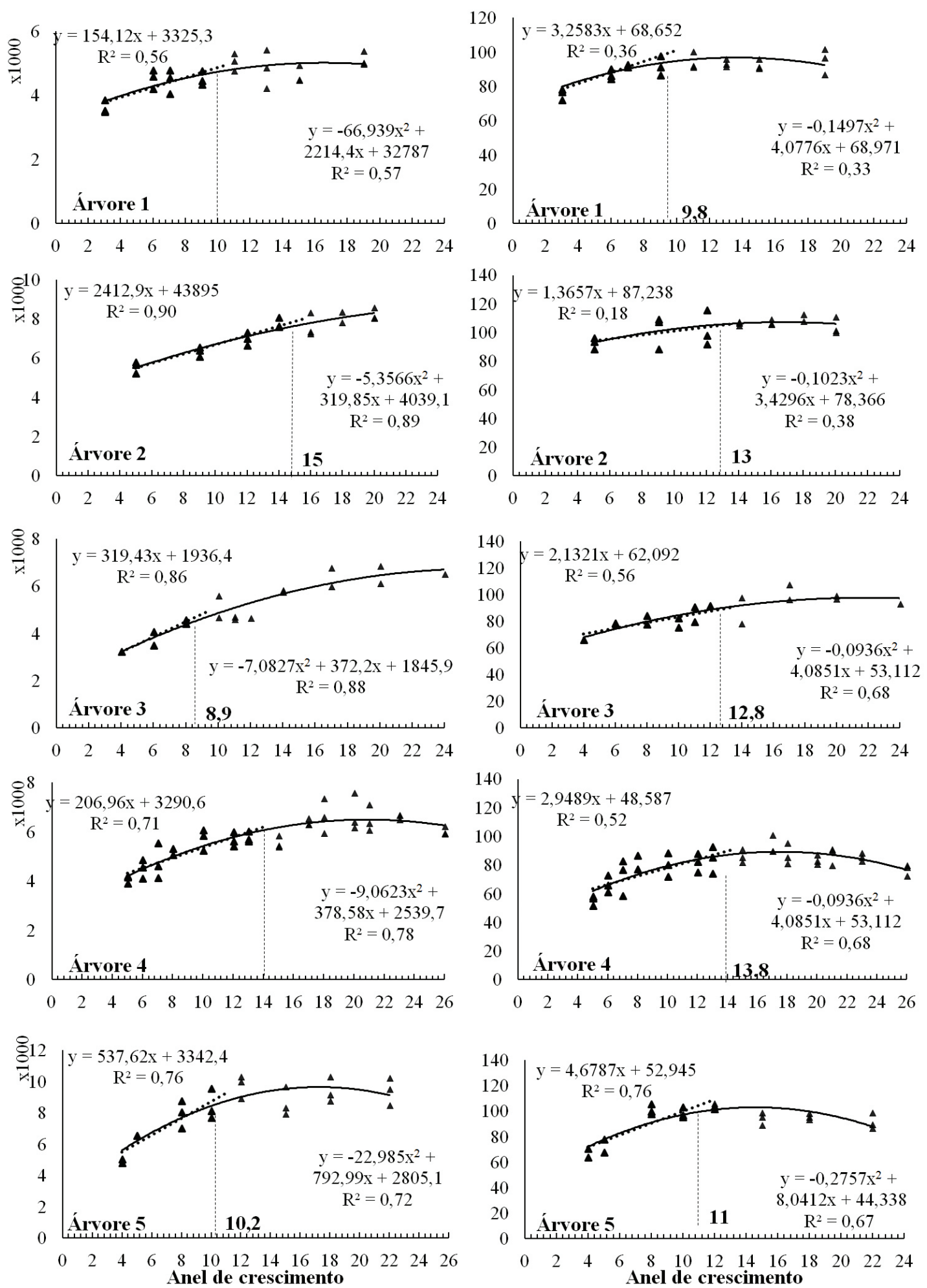

Figura 3. Variação do MOE (esquerda) e MOR (direita), e demarcação de lenhos juvenil e adulto para as cinco árvores de nogueira-pecã.

Figure 3. Variation of MOE (left) and MOR (right) and demarcation juvenile and mature zones for the five Nogueirapecã trees. 

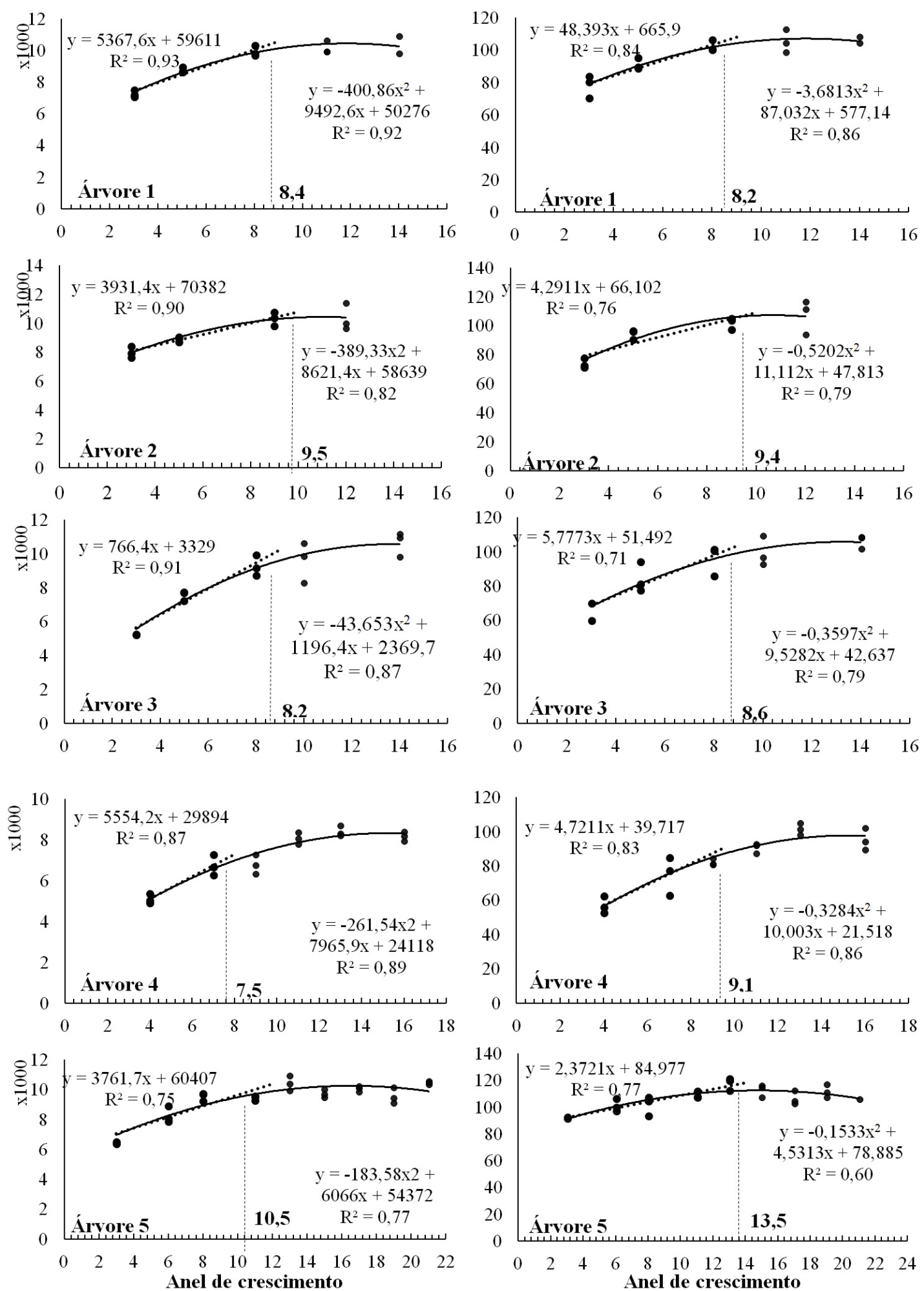

Figura 4. Variação do MOE (esquerda) e MOR (direita), e demarcação de lenhos juvenil e adulto para as cinco árvores de plátano.

Figure 4. Variation of MOE (left) and MOR (right) and demarcation juvenile and mature zones for the five Plátano trees. 
De acordo com a Tabela 2, constatou-se que não houve variação estatisticamente significativa para a idade de transição entre lenhos quando o fator Propriedade (MOE e MOR) é avaliado isoladamente, assim como a interação Espécie $\times$ Propriedade também não mostrou significância (Razão $F=0,87$ ). Por outro lado, o fator Espécie, quando avaliado isoladamente, mostrou significância, o que indica que pelo menos duas espécies apresentam idade de transição de lenhos diferentes estatisticamente.

Desconsiderando o fator não significativo (Propriedade, Razão F = 0,04), obteve-se idade média de transição entre lenhos de 9,3 para a madeira de plátano, 11,8 para nogueira-pecã e 23,8 para açoitacavalo.

Um comportamento muito similar ao verificado no presente estudo foi observado por Evans et al. (2000) para a madeira de Red alder (Alnus rubra), que observaram que a idade de transição do lenho juvenil para o adulto ocorreu entre 11 e 18 anos, quando utilizado o MOE e o MOR para estimar a delimitação. Os autores verificaram o mesmo tipo de comportamento polinomial para a variação do MOE e do MOR ao longo do sentido medula-casca.

Por outro lado, diversos estudos sobre a delimitação entre lenhos foram realizados por meio da variação das características anatômicas no sentido radial (Gatto et al., 2008, 2010; Leonello et al., 2008; Ferreira et al., 2011; Lobão et al., 2012) e mostraram que, por esses parâmetros, a idade de transição tende a ser mais tardia. Todavia, quando se utilizam propriedades como MOE, MOR ou massa específica, passa-se a avaliar a madeira como um material microestruturado e não apenas com características isoladas, tais como comprimento de fibras e espessura de parede. Assim, é possível que a diferença entre a idade de transição entre lenhos do presente estudo com outros anteriormente publicados deva-se a esse motivo, ou seja, a propriedade escolhida para a delimitação.

Outras espécies pesquisadas por Muñiz (1993) e Bendtsen (1978), por meio de propriedades físicas (massa específicas) e anatômicas (comprimento de fibras), apresentaram faixa de segregação do lenho entre o $5 .^{\circ}$ e o $20 .^{\circ}$ anel de crescimento, corroborando, dessa maneira, com os resultados avaliados neste trabalho.

Após a obtenção da idade de transição por meio do procedimento gráfico de regressões, os valores de MOE e MOR foram separados em lenho juvenil (abaixo da idade de transição) e lenho adulto (acima da idade de transição) e assim pôdese validar o método de delimitação de lenhos por meio da ANOVA fatorial apresentada na Tabela 3. Em seguida os parâmetros tidos como significativos foram desdobrados e avaliados separadamente, conforme se verifica na Tabela 4 .

Por meio da Tabela 3, verificou-se que o MOE varia de forma significativa em função da espécie e do lenho, quando avaliado isoladamente, ou seja, a interação entre os fatores não apresentou significância. Já para o MOR, as espécies e o tipo de lenho foram significativos de forma isolada e também quanto à interação.

Por meio do desmembramento da análise apresentado na Tabela 4, pode-se verificar que,

Tabela 3. Análise de variância fatorial para o MOE e o MOR em função da espécie e do lenho.

Table 3. Factorial analysis of variance for the MOE and MOR as a function of wood specie and juvenile and mature zones.

\begin{tabular}{cccccc}
\hline Propriedade & Fator & SQ & Gl & QM & Razão F \\
& Espécie (A) & $3,79 \times 10^{8}$ & 2 & $1,89 \times 10^{8}$ & $91,07^{\star *}$ \\
\cline { 2 - 4 } MOE & Lenho (B) & $1,01 \times 10^{8}$ & 1 & $1,01 \times 10^{8}$ & $48,65^{\star *}$ \\
& A $\times$ B & $8,46 \times 10^{6}$ & 2 & $4,23 \times 10^{6}$ & $2,03^{\text {ns }}$ \\
& Resíduo & $6,16 \times 10^{8}$ & 296 & $2,08 \times 10^{6}$ & \\
& Espécie (A) & 4988,08 & 2 & 2494,04 & $17,34^{\star *}$ \\
& Lenho (B) & 8304,34 & 1 & 8304,34 & $57,75^{\star *}$ \\
MOR & A $\times$ B & 1419,5 & 2 & 709,75 & $4,94^{\star *}$ \\
\hline
\end{tabular}

$\mathrm{SQ}=$ soma dos quadrados; $\mathrm{Gl}$ = grau de liberdade, $\mathrm{QM}=$ quadrado médio; ${ }^{* *}$ significativo em nível de $1 \%$ de probabilidade de erro; ns = não significativo. 
Tabela 4. Teste de média dos resultados tidos como significativos pela ANOVA fatorial.

Table 4. LSD Fisher test for the significant results by factorial ANOVA.

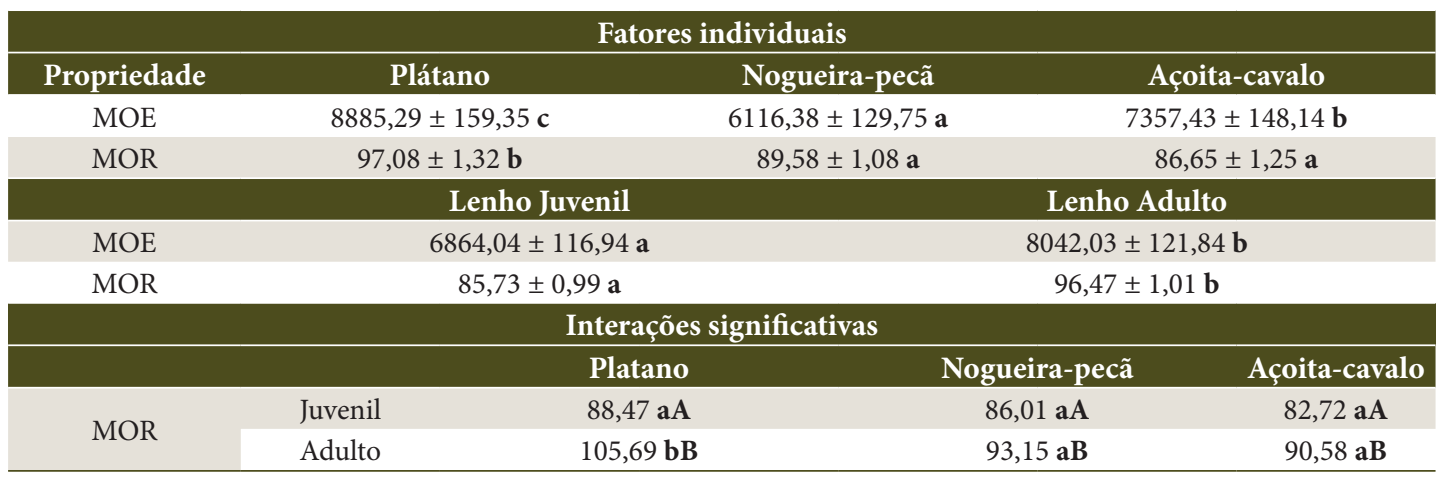

para o MOE, as três espécies apresentam resultados diferentes, sendo a madeira de plátano a de melhor resultado para as propriedades mecânicas e a madeira de nogueira-pecã, a de pior resultado para esse parâmetro. Para o MOR, a madeira de plátano mostrou resultados estatisticamente superiores quando comparada às outras duas espécies.

Quanto ao fator lenho avaliado isoladamente, pode-se observar que, tanto para o MOE como para o MOR, o lenho adulto apresentou resultados significativamente superiores ao lenho juvenil. $\mathrm{Na}$ interação (espécie $\times$ lenho) para o MOR, verificou-se que, em todas as situações, o lenho adulto apresenta resistência mecânica superior à do lenho juvenil. Nota-se que a diferença significativa para o MOE e o MOR entre os lenhos indicou que o procedimento de delimitação entre lenhos foi realizado com sucesso, pois, caso contrário, os resultados teriam apresentado igualdade estatística.

A maior resistência do lenho adulto quando comparado ao lenho juvenil de uma mesma espécie foi comprovada anteriormente por diversos autores, para diversas espécies florestais (Bao et al., 2001; Bhat et al., 2001; Passialis \& Kiriazakos, 2004; Adamopoulos et al., 2007; Gonçalves et al., 2009), corroborando, dessa forma, com os resultados obtidos no presente estudo.

\section{CONCLUSÕES}

A delimitação da zona de transição de lenhos juvenil e adulto para as madeiras de plátano, nogueira-pecã e açoita-cavalo foi realizada com sucesso e confirmada, posteriormente, por meio da análise de variância fatorial

Com base nos resultados, pode-se concluir que a madeira de melhor resistência mecânica para ambos os lenhos foi o plátano, assim como esta apresentou a idade de transição mais precoce, indicando que essa espécie apresenta madeira de boa qualidade mesmo em indivíduos jovens.

Para as três madeiras, o lenho juvenil apresentou resistência mecânica significativamente inferior ao lenho adulto.

Por fim, em relação ao comportamento do $\mathrm{MOE}$ e do MOR no sentido medula-casca, pode-se notar que a curva polinomial de segunda ordem serviu como o melhor ajuste para os dados.

\section{STATUS DA SUBMISSÃO}

Recebido: 10 out., 2012

Aceito: 18 mar., 2014

Publicado: 30 jun., 2014

\section{AUTOR(ES) PARA CORRESPONDÊNCIA}

\section{Bruno Dufau Mattos}

Centro de Desenvolvimento Tecnológico, Universidade Federal de Pelotas - UFPel, CEP 96010-000, Pelotas, RS, Brasil e-mail: brunodufaumattos@gmail.com

\section{APOIO FINANCEIRO}

Bolsa de Produtividade em Pesquisa II para o autor Prof. Dr. Darci Alberto Gatto. 


\section{REFERENNCIAS}

Adamopoulos S, Passialis C, Voulgaridis E. Strength properties of juvenile and mature wood in Black Locust (Robinia pseudoacacia L.). Wood and Fiber Science 2007; 39(2): 241-249.

American Society for Testing and Materials - ASTM. ASTM D 143-94: Standard methods of testing small clear specimens of timber. Philadelphia: ASTM; 2000.

Bao FC, Jiang ZH, Jiang XM, Lu XX, Luo XQ, Zhang SY. Differences in Wood properties between juvenile wood and mature wood in 10 species grown in China. Wood Science and Technology 2001; 35: 363-375. http://dx.doi. org/10.1007/s002260100099

Bendtsen BA. Properties of wood from improved and intensively managed trees. Forest Products Journal 1978; 28(10): 69-72.

Bhat KM, Priya PB, Rugmini P. Characterisation of juvenile wood in teak. Wood Science and Technology 2001; 34: 517-532. http://dx.doi.org/10.1007/ s002260000067

Comision Panamericana de Normas Técnicas - COPANT. Seleccion y colección de maderas. 1971. COPANT 30.

Evangelista WV, Silva JC, Della Lucia RM, Lobo LM, Souza MO. Propriedades físico-mecânicas da madeira de Eucalyptus urophylla S.T. Blake no sentido radial e longitudinal. Ciência da Madeira 2010; 1(2): 1-19. http://dx.doi.org/10.12953/2177-6830.v01n02a01

Evans JW, Senft JF, Green DW. Juvenile wood effect in Red alder: analysis of physical and mechanical data to delineate juvenile and mature wood zones. Forest Products Journal 2000; 50(7/8): 75-87.

Ferreira AL, Severo ETD, Calonego FW. Determination of fiber length and juvenile and mature wood zones from Hevea brasiliensis trees grown in Brazil. European Journal Wood Products 2011; 69: 659-662. http://dx.doi. org/10.1007/s00107-010-0510-2

Gatto DA, Haselein CR, Buligon EA, Calegari L, Stangerlin DM, Melo RR, et al. Estimativa da idade de segregação do lenho juvenil e adulto por meio de parâmetros anatômicos para madeira de Luehea divaricata Mart. Ciência Florestal 2008; 18(4): 535-540.

Gatto DA, Haselein CR, Buligon EA, Calegari L, Stangerlin DM, Melo RR, et al. Estimativa da idade de segregação do lenho juvenil e adulto de Carya illinoinensis (Wangenh) K. Koch por meio de parâmetros anatômicos da madeira. Ciência Florestal 2010; 20 (4): 6755-6682.
Gatto DA, Haselein CR, Buligon EA, Calegari L, Stangerlin DM, Oliveira LS. Estimativa da idade de segregação do lenho juvenil e adulto para platanus $\mathrm{x}$ acerifolia (Ait.) Willd. Revista Cerne 2007; 13 (4): 393398.

Gonçalves FG, Oliveira JTS, Della Lucia RM, Sartório RC. Estudo de algumas propriedades mecânicas da madeira de um hibrido clonal de Eucalyptus urophylla X Eucalyptus grandis. Revista Árvore 2009; 33(3): 501-509. http://dx.doi.org/10.1590/S0100-67622009000300012

Larson PR, Kretschmann DE, Clark A, Isebrands JG. Formation and properties of juvenile wood in southern pines: a synopsis. Forest Products Laboratory; 2001. General Technical Report.

Latorraca JVF, Albuquerque CEC. Efeito do rápido crescimento sobre as propriedades da madeira. Floresta e Ambiente 2000; 7 (1): 279-291.

Leonello EC, Palma HAL, Ballarin AW. Delimitação da madeira juvenil e adulta de Eucalyptus grandis em São Paulo, Brasil. Revista Forestal Venezolana 2008; 52(1): 93-98.

Lima IL, Garcia JN. Efeito da fertilização em propriedades mecânicas da madeira de Eucalyptus grandis. Ciência Florestal 2011; 21(3): 601-608.

Lobão MS, Costa DP, Almonacid MAA, Tomazello Filho M. Qualidade do lenho de árvores de Schizolobium parahyba VAR. amazonicum, Acre, Brasil. Floresta e Ambiente 2012; 19(3): 374-384. http://dx.doi. org/10.4322/floram.2012.044

Muñiz GLB. Caracterização e desenvolvimento de modelos para estimar as propriedades e o comportamento na secagem da madeira de Pinus taeda L. [tese]. Curitiba: Universidade Federal do Paraná; 1993.

O’Neill H, Tarigo F. Comportamiento en flexión de E. grandis, P.taeda y P. elliottii de madera de tamaño real $y$ de pequeñas probetas sin defectos. Montevideo: LATU; 2003. Informe de Investigacíon, 14.

Panshin AJ, Zeeuw C de. Textbook of wood technology: structure, identification, properties and uses of the commercial woods of the United States and Canada. New York: McGraw-Hill; 1964.

Passialis C, Kiriazakos A. Juvenile and mature wood properties of naturally-grown fir trees. Holz Roh Werkst 2004; 62: 476-478.

Zobel BJ, Buijtenen JP. Wood variation: its causes and control. Berlin: Springer Verlag; 1989. http://dx.doi. org/10.1007/978-3-642-74069-5 